\title{
CASTRAÇÃO PRÉ-PÚBERE E SUAS CONSEQQUÊNCIAS: revisão de literatura
}

\author{
Early neutering and its consequences: literature review
}

Larissa Rodrigues Marchini ${ }^{1}$, Ana Carolina de Andrade Leite de Camargo ${ }^{1}$

Lizandra Amoroso²

*Autor Correspondente: Larissa Rodrigues Marchini. Rua Augusto Coli, número 128, Jardim Alexandra Balbo, Sertãozinho, SP, Brasil. CEP: 14169-120.

E-mail: larissamarchini.fcav@gmail.com

\begin{abstract}
Como citar: MARCHINI, L. R.; CAMARGO, A. C. A. L.; AMOROSO, L. Castração pré-púbere e suas consequências: revisão de literatura. Revista de Educação Continuada em Medicina Veterinária e Zootecnia do CRMV-SP, São Paulo, v. 19, n. 1, 2021. DOl: https://doi.org/10.36440/recmvz.v19i1.38171.

Cite as: MARCHINI, L. R.; CAMARGO, A. C. A. L.; AMOROSO, L. Early neutering and its consequences: literature review. Journal of Continuing Education in Veterinary Medicine and Animal Science of CRMV-SP, São Paulo, v. 19, n. 1, 2021. DOl: https://doi.org/10.36440/recmvz.v19i1.38171.
\end{abstract}

\section{Resumo}

A cirurgia de castração é uma das técnicas cirúrgicas mais realizadas na rotina veterinária e a idade ideal para a sua execução ainda é contraditória entre os médicos-veterinários, uma vez que são escassos os estudos sobre este tema relacionados a alguns fatores, como raça e espécie. A castração é tida como método de contracepção, controle de zoonoses e método preventivo e terapêutico de algumas enfermidades como as afecções do sistema reprodutor. A castração pré-púbere é amplamente aplicada em diversos países, porém certas consequências desta prática, como o aumento do risco de aparecimento de algumas enfermidades como afecções urogenitais, obesidade, diabetes mellitus, afecções musculoesqueléticas e neoplasmas têm sido levantadas. Assim sendo, esta revisão de literatura pretende abordar a castração pré-púbere e suas consequências com o objetivo de contribuir para a tomada de decisão dos profissionais veterinários e de contribuir para a realização de estudos futuros.

Palavras-chave: Cães. Castração Pré-Pubere. Gatos. Riscos.

\section{Abstract}

Castration surgery is one of the most commonly performed surgical techniques in the veterinary routine, and the optimal age for its execution is still contradictory among veterinarians. Castration is considered a method of contraception, control of zoonoses and a preventive and

1 Discente da Faculdade de Ciências Agrárias e Veterinária, Universidade Estadual Paulista Júlio de Mesquita Filho, Jaboticabal, SP, Brasil

2 Docente da Faculdade de Ciências Agrárias e Veterinária, Universidade Estadual Paulista Júlio de Mesquita Filho, Jaboticabal, SP, Brasil 
therapeutic method for some diseases such as diseases of the reproductive tract. Early neutering is widely applied in several countries, but certain consequences of this practice, such as the increased risk of some diseases such as urogenital disorders, obesity, diabetes mellitus, musculoskeletal disorders and neoplasms must be considered. Therefore, this paper is a review of early neutering of dogs and cats and its consequences, in order to contribute to the decision-making of veterinary professionals, and collaborating with future studies.

Keywords: Dogs. Early Neutering. Cats. Scratches.

\section{Introdução: Definição, vantagens e prevalência}

A gonadectomia é um dos procedimentos mais rotineiros da cirurgia veterinária, e apesar de tradicional, é incomum encontrar dados que caracterizem a idade ideal para a sua realização de acordo com a espécie e padrão racial e se deve ser utilizada como cirurgia eletiva ou não (PALESTRINI, 2021). Atualmente, grande número de médicos-veterinários recomenda que a técnica deva ser executada em cães e gatos entre os seis e os nove meses de idade. No Brasil, a castração é frequentemente realizada após os seis meses, mas sabe-se que a idade adequada para a castração é muito variável e tem se modificado ao longo dos anos (KUSTRITZ, 2007), tornando-se um tema debatido entre médicosveterinários e pesquisadores (SERIN; ULUTAS, 2010). Alguns veterinários defendem a esterilização pediátrica, enquanto outros, o atraso na realização da técnica ou até mesmo a sua não realização (BUSHBY, 2018).

A castração é o método de contracepção recomendado para controle populacional de cães e gatos e diminuição do número de animais errantes (SERIN; ULUTAS, 2010). Mckenzie (2010) relatou que, nos EUA, no período compreendido entre os anos de 1970 a 2000, a prática rotineira da castração, em abrigos de cães determinou uma diminuição de 18,9 milhões de animais abandonados e que, possivelmente, seriam submetidos à eutanásia, o que ressalta a importância dessa prática para o bemestar animal.

Trevejo, Yang e Lund (2011) avaliaram a prevalência de castração nos Estado Unidos da América, de janeiro a dezembro de 2007 e constataram que de todos cães e gatos examinados em 651 hospitais privados do País, 262.912 (82,1\%) gatos e 861.300 (64\%) cães eram castrados. Na cidade de São Paulo, entre os anos de 2005 a 2008, a implantação de estratégias de controle populacional com o emprego da castração determinou uma diminuição de $8 \%$ e $18 \%$, respectivamente, nas taxas de natalidade canina e felina (GARCIA et al., 2018).

Gunther, Raz e Klement (2018) verificaram que, no período compreendido entre os anos de 2012 a 2014, na cidade de Rishon Lezion, em Israel, foi constatada a existência de associação entre castração com a saúde e o bem-estar da população de gatos que deambulavam livremente pelas ruas da cidade. De fato, de 4.615 animais observados, 2.365 eram adultos castrados, 1.445 adultos intactos e 692 filhotes. Os filhotes apresentaram maior prevalência de emaciação, incapacidade ou lesão grave que os adultos intactos.

Kreisler, Cornell e Levy (2019) realizaram análise retrospectiva de 1999 a 2013, na comunidade de Ocean Reef - Florida, do efeito do programa "trap-neuter-return", em que gatos itinerantes livres foram capturados, castrados, marcados, vacinados, vermifugados e liberados, constatando que a população de gatos livre registrada em 1999 diminuiu de 455 para 206 gatos em 2013, ou seja, houve $55 \%$ de redução. Ademais, o programa registrou 3.487 visitas, em que 2.529 eram gatos itinerários, sendo efetuadas 869 ovariohisterectomias e 822 orquiectomias. 0 programa foi benéfico reduzindo a população itinerante e aumentando a expectativa de vida e a sobrevida dos remanescentes.

Além da castração ser um recurso de controle populacional, ela também é um método importante para a prevenção de doenças associadas ao sistema geniturinário das fêmeas como: neoplasmas mamários, ovarianos e uterinos, piometra, hiperplasia endometrial cística, torção e prolapso uterino; já 
nos machos, a prática previne a hiperplasia benigna de próstata (HOWE, 2006). Além de prevenir doenças e controlar o tamanho da população, a castração também pode ocasionar mudanças comportamentais positivas, como docilidade e diminuição do hábito de marcar território (SERIN; ULUTAS, 2010).

A castração pré-púbere ou precoce, remoção cirúrgica das gônadas (gonadectomia), em idade pré-púbere é amplamente empregada em diversos países da Europa e nos Estados Unidos da América (ZAGO, 2013). No entanto, no Brasil, o procedimento ainda é um tema de muita polêmica no meio veterinário (SILVA, 2019). Isso porque há carência de dados sobre os protocolos anestésicos e técnicas cirúrgicas a serem empregados em pacientes pediátricos, considerando que, nessa fase, são mais propensos à hipotermia, hipoglicemia, paradas cardiorrespiratórias e sensibilidade à sobredose de fármacos (SILVA et al., 2015).

Kustritz (2002); Spain, Scarlett e Houpt (2004); e Reichler (2009) relataram que quando a castração é realizada precocemente, há aumento no risco do aparecimento de alterações urogenitais, obesidade, diabetes mellitus, distúrbios comportamentais, alterações em ossos longos, frouxidão ligamentar, lesões articulares e o desenvolvimento de neoplasmas.

Deste modo, o objetivo do presente trabalho é a análise crítica de algumas complicações e enfermidades que podem ocorrer em cães e gatos submetidos à castração precoce.

\section{Incontinência urinária, Doença do Trato Inferior dos Felinos e formação de estruturas do sistema geniturinário}

A incontinência urinaria é uma afecção, na maioria das vezes, incurável, que tem como sinais clínicos o acúmulo de urina no local que o animal adormece, e com a liberação de urina de forma involuntária quando o cão ou gato encontra-se em estado de excitação (JOHNSTON, 1991). A sua ocorrência é maior em fêmeas caninas castradas (4 a 21\%), quando comparadas a intactas (0,3\%), (REICHLER, 2009) e pode ocorrer por incompetência do esfíncter uretral ou por ser responsiva ao estrógeno (ALVES; HEBLING, 2020). 0 aumento do risco de desenvolvimento dessa afecção ainda é maior quando a técnica é realizada precocemente, antes dos três meses de idade, devido à imaturidade do sistema genital (HOWE, 2015). Isso ocorre porque, após o procedimento, há modificações fisiológicas, como alterações hormonais, redução da capacidade de contratilidade do músculo detrusor e de regulação da pressão de fechamento da uretra, o que pode provocar disfunção do óstio uretral (LICHTLER, 2014).

A síndrome denominada como Doença do Trato Urinário Inferior dos Felinos (DTUIF) é uma importante afecção que ocorre em felinos em que a incidência é aumentada após a castração. Os machos castrados precocemente são mais propensos a desenvolver DTUI e obstrução uretral, devido ao desenvolvimento incompleto do osso peniano e da uretra (JOYCE; YATES, 2011). Kustritz (2007) constatou que em ratos e coelhos precocemente castrados a privação androgênica determinou a ocorrência de alterações estruturais como a diminuição da área de tecido cavernoso e do músculo liso trabecular após o primeiro mês do procedimento,

A ausência de hormônios reprodutivos na fase pré-púbere pode ocasionar formação deficiente de órgãos reprodutores em cães e gatos (REICHLER, 2009). Em cadelas e gatas pode ocorrer má formação da genitália externa, involução do epitélio vaginal, diminuição do tamanho da vulva e maior predisposição a infeções como vaginites. Em cadelas, também há maior predisposição a dermatites perivulvares, especialmente se houver incontinência urinária (KUSTRITZ, 2007).

Stubbs (1996) constatou a ocorrência de prejuízos no desenvolvimento do osso peniano, pênis e prepúcio de cães machos, castrados com dois a três meses de idade. 
Gatos castrados precocemente apresentam desenvolvimento inadequado do osso peniano, atrofia ou ausência de espículas penianas e alteração na densidade de fibras elásticas e colágenas no pênis, reduzindo a complacência da região periuretral (BORGES et al., 2017).

As alterações nas estruturas do sistema geniturinário provocam dificuldade de micção e aumentam a frequência de obstruções urinárias. Além disso, o tecido que conecta o pênis ao prepúcio é androgéno-dependente e a carência precoce desses hormônios pode dificultar e até mesmo impedir a regressão desse tecido, o que impossibilita a exposição peniana nos animais (JOYCE; YATES, 2011).

\section{Obesidade e Diabetes mellitus}

A obesidade é uma doença metabólica inflamatória crônica comum em animais de companhia, e apresenta vários fatores predisponentes dentre eles a raça, idade, nutrição, estilo de vida e status reprodutivo (KUSTRITZ, 2007). Os hormônios sexuais estão intimamente relacionados à liberação de leptina pelo eixo hipotálamo-hipófise-adrenais. A leptina é responsável pelo controle de ingestão de alimentos e saciedade, e interage com o estrogênio no controle do apetite. Desta forma, como a castração diminui a concentração de hormônios sexuais, ela contribui para o desenvolvimento da obesidade por reduzir a produção de leptina pelo organismo (MELLO, 2011). Em humanos e animais a leptina é essencial para a prevenção da obesidade (HERMSDORFF; VIEIRA; MONTEIRO, 2006), pois modula o apetite e o balanço energético, uma vez que aumenta a expressão de neuropeptídios anorexígenos e inibe a formação de neuropeptídios orexígenos, além de aumentar o gasto energético para manter o peso corporal (NISWENDER; SCHWARTZ, 2003; ROMERO; ZANESCO, 2006). Desta forma, a castração interfere no controle de fome e saciedade, diminui a atividade física, e aumenta o ganho de peso de cães e gatos (SCHUSTER, 2017).

Kustritz (2002) observou que gatos castrados entre sete semanas e sete meses de idade apresentaram maior propensão ao ganho de peso e aumento de porcentagem de gordura quando comparados a felinos inteiros. Machado et al. (2017) investigaram a ingestão de alimentos póscastração em gatas. Um grupo era formado por fêmeas que ingeriram a mesma quantidade de alimento antes e pós-castração e um segundo grupo ingeriu quantidade superior à consumida antes da cirurgia. Os resultados mostraram que o grupo que continuou ingerindo a mesma quantidade de alimento ganhou peso pós-castração, o que indica que a necessidade energética desses animais foi alterada.

Além do aumento de consumo de alimentos, a castração em felinos eleva as concentrações de insulina plasmática, o que aumenta o risco de desenvolvimento de diabetes mellitus (DM) tipo II em 8,7 vezes se comparado a felinos inteiros (VOORWALD; TIOSSO; TONIOLLO, 2013). A DM tipo II tem grande prevalência nessa espécie, correspondendo a aproximadamente $90 \%$ dos casos de diabetes em gatos (RAND, 1999) e é altamente predisposta pela obesidade nesses animais (CISTERNAS, 2002). De fato, a leptina é produzida nos adipócitos, e quanto maior o número de adipócitos, maior o estímulo para a secreção de adipocinas (CARCIOFI, 2006), que além de participarem da regulação do metabolismo da gordura, têm importante função no metabolismo da glicose, bem como nas concentrações de insulina e glicose plasmáticas (CISTERNAS, 2002). Quando existem altas concentrações plasmáticas de glicose, há redução na sensibilidade do organismo à insulina, e a resistência à insulina determina a predisposição à ocorrência de síndromes metabólicas como a DM tipo II (SCOTT-MONCRIEFF, 2010).

\section{Afecções do sistema musculoesquelético em raças grandes e gigantes}

Durante o crescimento, os andrógenos e estrógenos exercem grande influência na forma e tamanho do esqueleto e na maturidade e contribuem para a homeostase óssea (MANOLAGAS; 
O'BRIEN; ALMEIDA, 2013). A castração pode estar associada ao aparecimento de enfermidades do sistema musculoesquelético, como a displasia coxofemoral (REICHLER, 2009). Alves e Hebling (2020) relataram que os hormônios gonadais podem influenciar no processo de fechamento dos ossos longos, e, portanto, a castração precoce pode predispor a essa alteração que é doença comum em cães e que está associada à dor no quadril, limitações funcionais e osteoartrite secundária. Em cães da raça boxer foi constatado que, nos animais castrados antes dos seis meses de idade, houve 1,5 vezes mais chances para o desenvolvimento de displasia coxofemoral quando comparados aos não castrados. No entanto, 0 peso corpóreo que é importante fator extrínseco modificador da displasia coxofemoral não foi incluído na análise (VAN HAGEN et al., 2005).

A displasia do cotovelo é afecção debilitante que acomete, principalmente, cães de raça grande a gigante (MICHELSEN, 2013).

Salmeri et al. (1991) avaliaram os efeitos da castração no crescimento do esqueleto de 32 cães machos e fêmeas sem raça definida distribuídos em três grupos: a) animais castrados com sete semanas de idade; b) castrados aos sete meses de idade e c) intactos. Relataram que a taxa de crescimento dos cães não foi afetada pela castração, mas o período de crescimento da metáfise distal da articulação radioulnar foi estendido em todos os cães machos, tanto nos castrados com sete semanas de idade, quanto aos sete meses de idade, e nas fêmeas castradas com sete semanas de idade.

A ruptura do ligamento cruzado cranial é uma das enfermidades articulares mais comuns em cães, causando a instabilidade no joelho, com consequente quadro de claudicação, degeneração articular progressiva e lesões meniscais secundárias (BRINKER, 1999). Ekenstedt et al. (2017) afirmaram que a causa definitiva da enfermidade não foi estabelecida, mas deve ser multifatorial (GUTHRIE et al., 2012). Spain, Scarlett e Houpt (2004) destacaram que o maior comprimento dos ossos longos de animais castrados precocemente altera o ângulo e a conformação das articulações, predispondo à ruptura do ligamento cruzado cranial. Hart et al. (2016) investigaram a associação entre a castração e o aparecimento de doenças articulares (displasia coxofemoral, ruptura do ligamento cruzado cranial e displasia do cotovelo) e concluíram que a ruptura do ligamento cruzado cranial foi a afecção que apresentou o maior aumento da incidência associada à castração precoce.

Ekenstedt et al. (2017) trabalharam com labradores retrievers e verificaram que, nas fêmeas castradas com um ano de idade ou menos, os casos de ruptura do ligamento cranial cruzado foram mais frequentes do que nas castradas com mais de um ano. Nos machos, a diferença entre os animais acometidos pela enfermidade e o grupo controle não foi significava. A conclusão obtida foi que a prática da castração antes de um ano de idade pode estar relacionada ao aumento de risco do desenvolvimento da afecção em labradores retrievers.

\section{Osteossarcoma em cães de raças de grande porte}

O osteossarcoma ou sarcoma osteogênico é o neoplasma ósseo primário mais diagnosticado no cão e o órgão mais frequentemente acometido por metástases é o pulmão (ANDRADE, 2009). Os animais de grande porte a gigantes são os mais acometidos (SELVARAJAH; KIRPENSTEIJN, 2010) e com idade média em torno de sete anos de idade (DALECK et al., 2006). Destaque-se, contudo, que os rottweilers, precocemente castrados, apresentam maior incidência do que outras raças (AKHTAR et al., 2015).

A etiologia do osteossarcoma canino é desconhecida, porém alguns autores afirmam ser multifatorial (MENDONÇA et al., 2008), entre as quais está incluída a castração (FIGUEIREDO, 2011). De fato, Belanger et al. (2017) afirmaram que cães castrados, machos ou fêmeas, têm maior risco de apresentar neoplasmas como osteossarcoma, mastocitoma e linfoma (BELANGER et al., 2017).

Nascimento et al. (2014) destacaram que em cães de ambos os sexos há importante participação do estrógeno na regulação óssea, pois os esteroides estimulam o aumento da formação do osso e inibem a reabsorção óssea e o estrógeno possui papel dominante nesse último caso. 
Howe (2015) analisando o desenvolvimento de lipoma em um amostra representativa da população de cães machos constatou a existência de risco praticamente nulo para cães castrados após doze meses de idade, enquanto a chance de desenvolvimento em cães castrados antes de um ano de idade foi de $98,6 \%$.

\section{Considerações finais}

A castração dos animais de companhia é considerada importante técnica de contracepção, controle populacional e de zoonoses, diminuição de cães e gatos errantes e um método preventivo ou terapêutico de diversas enfermidades. Aspectos individuais, fatores etiológicos, benefícios e riscos para a escolha do momento ideal do procedimento devem ser ponderados pelo médico-veterinário. 0 desenvolvimento musculoesquelético e geniturinário e a probabilidade de ocorrência de enfermidades em determinadas raças devem ser considerados na decisão da idade mais adequada para realização do procedimento cirúrgico. Os profissionais precisam avaliar os efeitos negativos da castração precoce em detrimento dos benefícios para a tomada de decisão. 0 médico-veterinário deve ter conduta profissional ética para minimizar prejuízos posteriores ao procedimento, assegurando, desta forma, segurança e qualidade de vida ao seu paciente.\&

\section{Referências bibliográficas}

AKHTAR, S. et al. A study investigating the epidemiological factors influencing the development of osteosarcoma in UK Rottweilers. BSAVA Congress Proceedings 2015, [s.l.], p. 442-442, 2015. DOI: https://doi.org/10.22233/9781910443521.57.6.

ALVES, B. F. A.; HEBLING, L. M. G. F. Vantagens e desvantagens da castração cirúrgica de cães domésticos: uma revisão integrativa de literatura. Brazilian Journal of Development, [s.I.], v. 6, n. 9, p. 73157-73168, 2020. DOl: https://doi.org/10.34117/bjdv6n9-683.

ANDRADE, S. A. F. Osteossarcoma canino: revisão de literatura. Revista UNILUS - Ensino e Pesquisa, [s.I.], v. 6, n. 10, p. 5-12, 2009.

BELANGER, J. M. et al. Correlation of neuter status and expression of heritable disorders. Canine genetics and epidemiology, [s.I.], v. 4, n. 1, p. 1-12, 2017. DOl: https://doi.org/10.1186/s40575-017-0044-6.

BORGES, N. C. et al. Effects of castration on penile extracellular matrix morphology in domestic cats. Journal of Feline Medicine and Surgery, [s.I.], v. 19, n. 12, p. 1261-1266, 2017. DOI: https://doi. org/10.1177/1098612X16689405.

BRINKER, W. O. A articulação fêmoro-tíbiopatelar (joelho). In: PIERMATTEI D. L, FLO, G. L. 3. ed. Manual de ortopedia e tratamento das fraturas dos pequenos animais. São Paulo: Manole, 1999. p. 480 - 538.

BUSHBY, P. A. The optimal age for spay/neuter: a critical analysis of spay neuter literature. In: SOUTHWEST VETERINARY SYMPOSIUM, 2018, Texas. Anais [...] Texas, 2018.

CARCIOFI, A. C. Obesidade e suas consequências metabólicas e inflamatórias em cães e gatos. Jaboticabal: Universidade Estadual Paulista, 2006. p. 7-12.

CISTERNAS, J. R. Fisiologia do tecido adiposo e leptina. In: DOUGLAS, C. R. Tratado de fisiologia aplicado à nutrição. São Paulo: Robe Editorial, 2002. p. 805.

DALECK, C. R. et al. Estudo retrospectivo de osteossarcoma primário dos ossos da pelve em cães em um período de 14 meses. Brazilian Journal of Veterinary Research and Animal Science, [s.l.], v. 43, n. 1, p. 125-131, 2006. DOI: https://doi.org/10.11606/issn.1678-4456.bjvras.2006.26527. 
EKENSTEDT, K. J. et al. Mutation status, sex, and sterilization status of a cohort of Labrador retrievers with and without cranial cruciate ligament rupture. Canine Genetics and Epidemiology, [s.I.], v. 4, n. 1, p. 2, 2017. DOI: https://doi.org/10.1186/s40575-017-0041-9.

FIGUEIREDO, M. S. Castração pré-púbere em cães e gatos - benefícios e riscos: revisão da literatura. 2011. 43 p. Trabalho de Conclusão de Residência - Escola de Veterinária. Universidade Federal de Minas Gerais, Belo Horizonte, 2011.

GARCIA, R. et al. Dog and cat population dynamics in an urban area: evaluation of a birth control strategy. Pesquisa Veterinária Brasileira, [s.I.], v. 38, n. 3, p. 511-518, 2018. D0I: https://doi. org/10.1590/1678-5150-PVB-4205.

GUNTHER, I.; RAZ, T.; KLEMENT, E. E. Association of neutering with health and welfare of urban freeroaming cat population in Israel, during 2012-2014. Preventive Veterinary Medicine, [s.I.], v. 157, p. 26-33, 2018. DOI: https://doi.org/10.1016/j.prevetmed.2018.05.018.

GUTHRIE, J. W. et al. Effect of signalment on the presentation of canine patients suffering from cranial cruciate ligament disease. Journal of Small Animal Practice, [s.I.], v. 53, n. 5, p. 273-277, 2012. DOI: https://doi.org/10.1111/j.1748-5827.2011.01202.x.

HART, B. L. et al. Neutering of German Shepherd Dogs: associated joint disorders, cancers and urinary incontinence. Veterinary Medicine and Science, [s.I.], v. 2, n. 3, p. 191-199, 2016. DOI: https://doi. org/10.1002/vms3.34.

HERMSDORFF, H. H. M.; VIEIRA, M. A. D. Q. M.; MONTEIRO, J. B. R. Leptina e sua influência na patofisiologia de distúrbios alimentares. Revista de Nutrição, [s.I.], v. 19, n. 3, p. 369-379, 2006. D0I: https://doi.org/10.1590/S1415-52732006000300008.

HOWE, L. M. Current perspectives on the optimal age to spay/castrate dogs and cats. Veterinary Medicine: Research and Reports, [s.l.], v. 6, p. 171, 2015. DOl: https://doi.org/10.2147/VMRR.S53264. HOWE, L. M. Surgical methods of contraception and sterilization. Theriogenology, [s.I.], v. 66, n. 3, p. 500-509, 2006. DOI: https://doi.org/10.1016/j.theriogenology.2006.04.005.

JOHNSTON, S. D. Questions and answers on the effects of surgically neutering dogs and cats. Journal of the American Veterinary Medical Association, [s.I.], v. 198, n. 7, p. 1206-1214, 1991.

JOYCE, A.; YATES, D. Help stop teenage pregnancy!: early-age neutering in cats. Journal of Feline Medicine and Surgery, [s.I.], v. 13, n. 1, p. 3-10, 2011. DOl: https://doi.org/10.1016/j. jfms.2010.11.005.

KREISLER, R. E.; CORNELL, H. N.; LEVY, J. K. Decrease in population and increase in welfare of community cats in a twenty-three year trap-neuter-return program in Key Largo, FL: the ORCAT Program. Frontiers in veterinary science, [s.I.], v. 6, p. 7, 2019. DOl: https://doi.org/10.3389/ fvets.2019.00007.

KUSTRITZ, M. V. R. Determining the optimal age for gonadectomy of dogs and cats. Journal of the American Veterinary Medical Association, [s.I.], v. 231, n. 11, p. 1665- 1675, 2007. DOI: https://doi. org/10.2460/javma.231.11.1665.

KUSTRITZ, M. V. R. Early spay-neuter: clinical considerations. Clinical Techniques in Small Animal Practice, [s.I.], v. 17, n. 3, p. 124-128, 2002. DOI: https://doi.org/10.1053/svms.2002.34328.

LICHTLER, J. Castração precoce em pequenos animais: técnica, vantagens e riscos e uso no controle populacional. 2014. 72 p. Trabalho de Conclusão de Curso - Faculdade de Veterinária, Universidade Federal do Rio Grande do Sul, Porto Alegre, 2014.

MACHADO, G. S. et al. Monitoring energy requirement and weight gain in adult cats after ovariectomy. World, [s.l.], v. 7, n. 2, p. 36-39, 2017. 
MANOLAGAS, S. C.; O'BRIEN, C. A.; ALMEIDA, M. The role of estrogen and androgen receptors in bone health and disease. Nature Reviews Endocrinology, [s.I.], v. 9, n. 12, p. 699, 2013. DOI: http://dx.doi. org/10.1038/nrendo.2013.179.

MCKENZIE, B. Evaluating the benefits and risks of neutering dogs and cats. CAB Reviews, [s.l.], v. 5, n. 45, p.1-18, 2010. DOI: http://dx.doi.org/10.1079/PAVSNNR20105045.

MELLO, W. G. D. Efeitos da castração e androgenização neonatal sobre o dimorfismo sexual esquelético e secreção de leptina e corticosterona em ratos. 2011. 54 p. Dissertação (Mestrado) Faculdade de Odontologia de Araçatuba, Universidade Estadual Paulista, Araçatuba, 2011.

MENDONÇA, F. et al. Osteosarcoma vertebral em cão-relato de caso. Revista Clínica Veterinária, [s.I.], v. 76, p. 48-52, 2008.

MICHELSEN, J. Canine elbow dysplasia: aetiopathogenesis and current treatment recommendations. The Veterinary Journal, [s.I.], v. 196, n. 1, p. 12-19, 2013. DOl: http://dx.doi.org/10.1016/j. tvjl.2012.11.009.

NASCIMENTO, O. B. et al. Efeitos dos esteróides sexuais sobre metabolismo ósseo: uma revisão de literatura. Revista de Odontologia da UNESP, [s.l.], v. 43, n. especial, 2014.

NISWENDER, K. D.; SCHWARTZ, M. W. Insulin and leptin revisited: adiposity signals with overlapping physiological and intracellular signaling capabilities. Frontiers in neuroendocrinology, [s.l.], v. 24, n. 1, p. 1-10, 2003. DOl: http://dx.doi.org/10.1016/s0091-3022(02)00105-x.

PALESTRINI, C. et al. Influence of gonadectomy on canine behavior. Animals, [s.I.], v. 11, n. 2, p. 553, 2021. DOI: http://dx.doi.org/10.3390/ani11020553.

RAND, J. Current understanding of feline diabetes mellitus: part 1, pathogenesis. Journal Feline Medicine Surgery, [s.I.], v. 1, p. 143-53, 1999. DOl: http://dx.doi.org/10.1016/S1098-612X(99)90203-6.

REICHLER, I. M. Gonadectomy in cats and dogs: a review of risks and benefits. Reproduction in Domestic Animals, [s.l.], v. 44, p. 29-35, 2009. DOl: http://dx.doi.org/10.1111/j.1439-0531.2009.01437.x.

ROMERO, C., ZANESCO, A. O papel dos hormônios leptina e grelina na gênese da obesidade.

Revista de Nutrição, [s.I.], v. 19. n. 1, p. 85-91, 2006. DOI: https://doi.org/10.1590/S1415$\underline{52732006000100009 .}$.

SALMERI, K. R. et al. Gonadectomy in immature dogs: effects on skeletal, physical, and behavioral development. Journal of the American Veterinary Medical Association, [s.I.], v. 198, n. 7, p. 11931203, 1991.

SCHUSTER, L. A. H. Efeitos da castração sobre o ganho de peso e a atividade física em cadelas. 2017. 50 p. Dissertação (Mestrado) - Faculdade de Veterinária, Universidade Federal do Rio Grande do Sul, Porto Alegre, 2017.

SCOTT-MONCRIEFF, J. C. Insulin resistance in cats. Veterinary Clinics of North America: Small Animal Practice, [s.l.], v. 40, n. 2, p. 241-257, 2010. DOI: https://doi.org/10.1016/j.cvsm.2009.10.007.

SELVARAJAH, G. T.; KIRPENSTEIJN, J. Prognostic and predictive biomarkers of canine osteosarcoma. The Veterinary Journal, [s.l.], v. 185, n. 1, p. 28-35, 2010. DOl: https://doi.org/10.1016/j. tvjl.2010.04.010.

SERIN, G.; ULUTAS, P. A. Measurement of serum acute phase proteins to monitor postoperative recovery in anoestrous bitches after ovariohysterectomy. Veterinary Record, [s.I.], v. 166, n. 1, p. 2022, 2010. DOI: https://doi.org/10.1136/vr.b5585.

SILVA, T. C. Castração pediátrica e não pediátrica em cães e gatos: resultados a longo prazo para saúde e comportamento dos animais. 2019, 136 p. Tese (Doutorado) - Universidade Federal Rural de Pernambuco, Recife, 2019. 
SILVA, T. C. et al. Conhecimento e percepção dos médicos-veterinários do hospital veterinário da UFRPE sobre a castração pediátrica. Revista de Educação Continuada em Medicina Veterinária e Zootecnia do CRMV-SP, São Paulo, v. 13, n. 3, p. 72-72, 2015.

SPAIN, C. V.; SCARLETT, J. M.; HOUPT, K. A. Long-term risks and benefits of early-age gonadectomy in dogs. Journal of the American Veterinary Medical Association, [s.I.], v. 224, n. 3, p. 380-387, 2004. DOI: https://doi.org/10.2460/javma.2004.224.380.

STUBBS, W. P. et al. Effects of prepubertal gonadectomy on physical and behavioral development in cats. Journal of the American Veterinary Medical Association, [s.I.], v. 209, n. 11, p. 1864, 1996.

TREVEJO, R.; YANG, M.; LUND, E. M. Epidemiology of surgical castration of dogs and cats in the United States. Journal of the American Veterinary Medical Association, [s.I.], v. 238, n. 7, p. 898-904, 2011. DOl: https://doi.org/10.2460/javma.238.7.898.

VAN HAGEN, M. A. et al. Incidence, risk factors, and heritability estimates of hind limb lameness caused by hip dysplasia in a birth cohort of Boxers. American Journal of Veterinary Research, [s.l.], v. 66, n. 2, p. 307-312, 2005. DOI: https://doi.org/10.2460/ajvr.2005.66.307.

VOORWALD, F. A.; TIOSSO, C. D. F.; TONIOLLO, G. H. Gonadectomia pré-puberal em cães e gatos. Ciência Rural, [s.I.], v. 43, n. 6, p. 1082-1091, 2013. DOl: https://doi.org/10.1590/S0103$\underline{84782013005000059}$.

ZAGO, B. S. Prós e contras da castração precoce em pequenos animais. 2013. 30 p. Trabalho de Conclusão de Curso - Faculdade de Veterinária, Universidade Federal do Rio Grande do Sul, Porto Alegre, 2013. 